\title{
Publisher's Note: Model-based optimization of tapered free-electron lasers [Phys. Rev. ST Accel. Beams 18, 040702 (2015)]
}

Alan Mak, Francesca Curbis, and Sverker Werin

(Received 3 November 2017; published 17 November 2017)

DOI: 10.1103/PhysRevAccelBeams.20.119902

This paper was published online on 23 April 2015 with an omission of an Acknowledgment section, as well as typographical errors in Eq. (2) and the caption to Fig. 1.

Equation (2) should read as

$$
a_{w}=\frac{K}{\sqrt{2}} \equiv \frac{e \lambda_{w} B_{w}}{2 \sqrt{2} \pi m_{e} c}
$$

The third line of the caption to Fig. 1 should read as "... trapping fraction, and (f) the bunching factor. The ...." The Acknowledgment section should be placed on page 11 and read as "The simulations presented in this article were performed on resources provided by the Swedish National Infrastructure for Computing (SNIC) at Lund University." The paper has been corrected as of 10 November 2017. 